\title{
MEMÓRIA: UM DESAFIO À AUTONOMIA DO IDOSO
}

\author{
MEMORY: A CHALLENGE TO THE AUTONOMY OF THE ELDERLY
}

Ana Lucia Fiebrantz Pinto ${ }^{1}$

RESUMO: 0 estudo das funções cognitivas é uma área em franco desenvolvimento no campo da neurociência, e de interesse geral. Estudos e pesquisas procuram caracterizar os problemas cognitivos e diferenciar o normal do patológico. Verifica-se que muitos idosos queixam-se de dificuldades de memória e que algum declínio da função cognitiva pode ser observada no processo de envelhecimento normal, fato que preocupa o idoso e a família pois geralmente é associado à ideia de dependência. 0 esquecimento deve ser diferenciado da "perda da memória" associada a processos demenciais. As ciências avançam neste sentido e, ressalte-se em especial a contribuição da neuropsicologia. Estudos demonstram a existência de patologias invalidantes acompanhadas de déficit importante da função da memória com perda de autonomia e independência. Há que se salientar, no entanto, a relação da memória com o contexto bio-psic-social do indivíduo. A memória é viva, trabalha e muda permanentemente.

PALAVRAS CHAVE: Memória; Envelhecimento; Autonomia; Família.

\section{INTRODUÇÃO}

No trabalho profissional cotidiano observa-se que o "esquecimento", "as falhas da memória" são as queixas mais freqüentes entre as pessoas que envelhecem. Esta queixa, em geral, é acompanhada por uma preocupação subjacente, na maioria das vezes oriundas da família e nem sempre declarada pelo paciente na primeira entrevista. Logo que se adquire confiança ou que se atenue o risco de uma causa grave estar associada a alteração da memória, descobre-se que o grande medo seria a perda da autonomia e o risco da dependência.

Outras queixas da memória como o "branco ocasional", equivalente a não se lembrar onde guardou os óculos ou as chaves, esquecer o nome de uma pessoa conhecida que não se vê há algum tempo, são ocorrências comuns a todas as pessoas. Até uma certa idade, considera-se este fato uma distração. De uma hora para outra, isto pode ser sentido como um sinal de senilidade inexorável com repercussões emocionais sérias atingindo também, o equilíbrio da família do idoso (Guillet, 1989).

U ma série de "fantasmas" advém da preocupação com a "falha da memória". Entre eles, o medo de não ser apto a compreender o que é dito, de não se lembrar de coisas importantes, de não poder se comunicar com outras pessoas, de não ser capaz de se cuidar e, em última instância, de se tornar "demente".

Com o passar do tempo, os reflexos das "falhas de memória", "dos pequenos e acumulados esquecimentos" na vida quotidiana, somados aos efeitos causados no meio familiar e no grupo de amigos, fazem surgir no indivíduo o sentimento de um "futuro desastroso" (G uillet, 1989).

Atualmente é muito comentado em todos os meios, a ocorrência de degeneração cerebral, déficit intelectual, declínio cognitivo, doença de Alzheimer... e, a alteração da memória como o sintoma mais enfatizado. Por esta razão, todo esquecimento é interpretado como algo grave pela população geral, uma vez que esta informação vem associada ao envelhecimento. Existe, no senso comum, uma idéia equivocada de que a velhice é uma etapa da vida que evolui apenas com perdas, uma sequência de problemas e dependência. Este fato ocorre por não se fazer distinção entre velhice e doença e, no caso, de alterações da memória com o início de um processo demencial.

Enfermeira, Psicóloga, Especialista em Gerontologia, Diretora da Área de Gerontologia da Fundação de A poio e Valorização do Idoso; Vice-presidente da Associação Nacional de Gerontologia. 
Os estudos epidemiológicos demonstram que a prevalência das demências na comunidade geral indicam uma variação de 1 a 2\% nas pessoas acima de 60 anos e de 3 a 9\% entre as pessoas de 65 anos - a demência caracteriza-se como doença (Guirlland, 1982). Outros trabalhos desenvolvidos nesta área de investigação evidenciam a presença de déficit cognitivo associado ao processo de envelhecimento normal.

A angústia na busca de uma explicação para o esquecimento e, de uma solução para o "problema", torna-se presente na maioria das pessoas que envelhecem mas passa a ser uma preocupação constante para os familiares.

A relação estabelecida entre velhice e dependência é uma representação antagônica ao ideal humano que é entre outros, o de manter-se ativo, produtivo, lúcido, saudável e ser capaz de tomar suas próprias decisões. "Não querer incomodar os outros" nas palavras dos próprios idosos. Há que se diferenciar o que é envelhecimento saudável e doença no envelhecimento. A doença pode acarretar sim, um processo de dependência e perda de autonomia, não a velhice em si mesma (Salazar, 1990).

É necessário buscar meios para garantir à pessoa que envelhece as condições para se manter ativo, independente e autônomo, aspiração maior de quem alcança a velhice.

As queixas e preocupações relativas à memória, sua relação com o envelhecimento e autonomia, não são um tipo de dificuldade individual, pois, é uma questão que se coloca ao homem, a vida, à nossa civilização, ao nosso tempo.

\section{A MEMÓRIA NO PROCESSO DE ENVELHECIMENTO}

Pode-se entender um pouco melhor as preocupações com a memória, quando se verifica que ao longo dos séculos as representações da cabeça sempre foram ligadas às expressões como: inteligência, razão, controle, caráter, lucidez e direção.

Estas concepções do senso comum mostram também que a cabeça é mais do que a caixa craniana e a massa nervosa alojada nela. A palavra cabeça designa ao mesmo tempo o que emana dela o que pode ser denominado também de pensamento, inteligência, personalidade (R otrou, 1993).

Quando se fala em memória de um modo geral, se entende o mecanismo de registro, consolidação e restituição no tratamento de informações. Para que essas operações ocorram, são necessárias outras funções associadas à memória. Estas funções são denominadas de funções cognitivas. Diz-se: "incógnito", aquilo que é desconhecido, portanto, "cognitivo", o que é conhecido e, cognição se refere à faculdade de adquirir conhecimento (Van der Linden, 1994).

Relativo a "cabeça" e o envelhecimento, observa-se que este pode acarretar declínios das funções cognitivas adquiridas, e que estão sendo estudados no sentido de diferenciar o que é normal do patológico, principalmente na área da neurociência e da gerontologia: "Ciência do Envelhecimento" (Canineu, 1997).

As funções cognitivas desempenham um papel importante no processo de aprendizagem e na vida das pessoas. Todos os comportamentos são decorrentes de aprendizagem, das mais elementares às mais complexas cujo processo prepara o homem, ao longo da vida, para o auto-cuidado, autonomia e independência. As funções cognitivas são primordiais para que isso possa ocorrer. As principais funções cognitivas são: atenção, concentração, percepção, linguagem, memória e inteligência. Estas funções todas dependem de um substrato biológico, são o resultado dos processos neurofisiológicos e bioquímicos cerebral (Souza, 1996).

Sabe-se que estas funções começam a apresentar um certo declínio à partir da terceira década da vida e este, prossegue no curso do envelhecimento. Há aquelas que são mais preservadas, entre elas o vocabulário. A função verbal, por exemplo, parece não se alterar até os sessenta anos. A reserva de informações a longo prazo se mantém mais preservada. As mais alteradas são principalmente as que envolvem execução (Bottino et al, 1997).

Folstein (1975) realizou um estudo com 923 (novecentos e vinte três) idosos com 65 (sessenta e cinco) anos ou mais, no qual utilizou um instrumento elaborado por ele próprio denominado (Mini Mental State), para investigar a presença de déficit cognitivo. O Mini Exame do Estado Mental, utilizado até hoje por vários profissionais da área da saúde e Gerontologia, entre eles também enfermeiros, possibilita detectar sinais de alterações da memória e é usado como um instrumento auxiliar no diagnóstico clínico.

Outros instrumentos técnicos de avaliação neuropsicológica tem sido utilizados para investigar as mudanças das funções cognitivas .Tarefa difícil, pois, nos estágios iniciais as alterações cognitivas relativas à 
demência se assemelham ao declínio cognitivo normal. Outros fatores contribuem para a modificação na cognição, entre eles: causas psiquiátricas, doenças orgânicas, isolamento social, farmacoterapia e drogas, induindo-se o "álcool" (Almeida, 1997).

$\mathrm{Na}$ idade avançada os declínios cognitivos guardam também uma relação estreita com fatores psicossociais e afetivos como: perdas de familiares ou amigos próximos, perda de postos ou cargos no trabalho, situações traumáticas na família. Este fato pode ser observado na avaliação clínica e no desempenho de pessoas submetidas a testes neuropsicológicos que têm valor importante no diagnóstico diferencial em estados depressivos, por exemplo. É preciso considerar também que o desempenho e o resultado apresentado nestes testes estão relacionados aos diferentes níveis socio-econômico e cultural (Gilmore, 1974). Por esta razão deveremos ter muito cuidado na utilização e dimensão dada aos resultados neles obtidos.

$\mathrm{Na}$ avaliação da memória é importante considerar que os testes são um dos fatores que devem ser utilizados na metodologia de avaliação do estado cognitivo e que não substitui nenhum outro mas, deve ser considerado como mais um recurso diagnóstico.

0 estudo da memória tem permitido que os autores identifiquem características diferentes e que classifiquem a memória de diferentes maneiras .

Segundo Rotrou (1993), possuímos três tipos de memória:

- A memória genética, informações contidas nos gens;

- A memória cultural, constituída de usos e costumes, o que foi adquirido.

- A memória transacional, as informações acumuladas durante a vida do indivíduo. Este é o tipo de memória que as pessoas comumente definem como memória.

0 processo de aprendizagem esta estreitamente associado ao processo de memória. Mattos (1999), em palestra, num encontro de Envelhecimento Cerebral, relata que muitos dos problemas cognitivos detectados na avaliação neuropsicológica para avaliação cognitiva no idoso, podem ser resultantes de distúrbios de aprendizagem não diagnosticados na infância.

Estudos e pesquisas procuram esclarecer o funcionamento da memória. Quanto mais avançam e se aprofundam, mais se verifica que sabe-se muito pouco e é preciso continuar estudando esta função cognitiva e, em especial a sua relação com o envelhecimento.

As questões mais freqüentes a respeito da memória são as relativas à sua localização e funcionamento e, mais recentemente, às associadas ao envelhecimento e as consequências sociais e familiares. Associações científicas, cientistas, técnicos e políticos se envolvem cada vez mais no interesse deste assunto dada a repercussão do fenômeno numa sociedade que envelhece.

Historicamente os romanos imaginavam que a memória era como uma rede de conectores complexa ligados entre si. Posteriormente, acreditava-se que era como um lugar onde se guardavam as lembranças. Com o avanço tecnológico as máquinas passaram a ser o paradigma. A memória já foi comparada à uma central telefónica e, mais recentemente ao computador.

$\mathrm{Na}$ tentativa de compreender a memória e seu funcionamento, o homem segue sua busca e, como observa Van Eersel (1986): "Os homens tem sempre tentado comparar o cérebro à tecnologia mais sofisticada da sua época (...). Em realidade, este pequeno jogo não esta perto de acabar e se compara o cérebro a muitas máquinas ainda (...). Como se as produções cerebrais (as máquinas) deveriam sempre servir de espelho à nosso próprio funcionamento interno, numa fuga sem fim. Nosso cérebro poderia conceber qualquer coisa que seja complexa assim como ele próprio?"

A evolução da nossa espécie levou milhões de anos para que nosso cérebro tivesse a composição atual. $\mathrm{Na}$ evolução filogenética o cérebro inicialmente compreendia apenas a parte central, depois sistema límbico e, finalmente, os hemisférios cerebrais (Barbizet et al, 1985).

Órgão complexo por natureza, constituído pelas células denominadas de neurônios que, mesmo diferentes em forma e tamanho, têm características comuns. A estrutura do neurônio é composta por um corpo celular, que contém o núcleo e o citoplasma e, pelos axônios e dendritos. 0 seu funcionamento ficou mais claro com a evolução dos estudos sobre o cérebro e o sistema nervoso (F aria, 1996).

0 cérebro do homem possui dois hemisférios: o direito e o esquerdo, os quais são ligados por feixes de fibras nervosas cruzadas: o corpo caloso. 0 corpo caloso garante a integração entre as diferentes zonas de cada um dos hemisférios. Os hemisférios cerebrais não possuem exatamente as mesmas funções. Considera- 
se que o hemisfério direito é mais especializado na gestão do espaço, na criatividade, nas artes plásticas e na afetividade e, o hemisfério esquerdo se relaciona às atividades verbais, gestuais, raciocínio lógico. A especialização entre os hemisférios é apenas uma questão de grau, e varia de pessoa para pessoa (Penfield e Roberts, 1959 in Tarnopol, 1980).

Recobrindo os dois hemisférios temos: a córtex cerebral. Nesta região estão as funções cerebrais mais evoluídas. As funções específicas, estão localizadas nos quatro lobos cerebrais. Os lobos occipitais se relacionam com a percepção e tratamento das informações visuais. Os lobos parietais tratam as informações sensitivas. Os lobos temporais são consideradas responsáveis pelas informações audioverbais. Os lobos frontais geradores de decisões, ações e capacidade de abstração.

Por contribuição da neuropsicologia, sabe-se que em cada lobo localiza-se uma zona primária, sensorial e motora, envolvidas por pequenas zonas denominadas "associativas". Estas tem papel preponderante nas funções superiores. Os estudos de Lúria (1981) foram fundamentais para a compreensão funcional dos sistemas cerebrais.

A localização cerebral permite uma relação topográfica do comportamento. No caso, por exemplo de um acidente com traumatismo no lobo frontal esquerdo, se a pessoa for destra, ela poderá ter como conseqüência uma afasia. Contudo, esta aparente simplicidade leva ao risco de se reduzir muito a complexidade do cérebro (Rotrou, 1993).

"Para tentar compreender atos comportamentais em termos de atividades cerebral, Lúria em 1966 desenvolveu uma abordagem denominada localização dinâmica das funções corticais" (Tarnopel, 1980).

A compreensão do funcionamento cerebral evoluiu mais quando "passou-se (...) a acreditar que a transmissão de informações entre neurônios ocorre principalmente em sítios de contato morfologicamente diferenciados, as sinapses, através da liberação de uma substância química - o neurotransmissor" (G raeff e Brandão 1997).

0 estudo dos neurotransmissores permitiu a evolução no entendimento dos mecanismos cerebrais envolvidos nos processos mentais. A identificação de suas vias permitem a localização de certos problemas mentais e, as alterações na produção destes neurotransmissores já podem ser corrigidas, facilitando o tratamento de algumas doenças, o que anteriormente era impossível. Exemplo clássico, o da Doença de Parkinson, relacionada com a dopamina (Brandão, 1997; Teive e Meneses, 1996).

No que se refere a memória, várias pesquisas e descobertas vem sendo feitas mas, pouco se sabe sobre o seu mecanismo. Descobriu-se, no entanto, que a via que inerva o hipocampo pode mediar processos da memória e a via que segue para a amígdala pode estar envolvida na modulação de processos afetivos. Estes estudos têm contribuído para o desenvolvimento de medidas terapêuticas em neuropatologia. A localização das vias permite saber que na demência tipo Alzheimer ocorre uma redução considerável das enzimas que sintetizam a acetilcolina no prosencéfalo basal e, que o núcleo basal de Maynert sofre uma degeneração acentuada e seletiva (Brandão, 1997), muito embora, não se tenha até o momento um tratamento efetivo para este mal.

Estas descobertas contribuem para fazer avançar os caminhos da ciência. Mas, está longe a mudança da vida cotidiana dos indivíduos que apresentam um declínio patológico das funções cognitivas e das famílias de portadores. É sobre estas que recaem as responsabilidades diretas e indiretas decorrentes da doença, tornando-se um problema bio-psico-social com reflexos familiares importantes, chegando até causar desestruturação de seus membros e do grupo.

Do ponto de vista biológico e funcional, verifica-se que o lobo temporal apresenta uma relação importante com a memória na função de armazenamento de eventos passados.

Ao neo-córtex temporal parece estar relacionado a memória de longo prazo. Na região do hipocampo as estruturas corticais que 0 circundam e as vias que ligam estas estruturas com outras partes do cérebro parecem exercer a função da memória para fatos e eventos. 0 hipocampo esta ligado com as relações espaciais, com a seleção de localização para o armazenamento de fatos e eventos, com o reconhecimento de novidades. Os estímulos sensoriais oriundos do meio externo, tais como: cheiro, sabor, visualização, som, sensação de objetos são transformados em sinais elétricos que ativam um circuito na amígdala que está relacionado com a memória, o qual depende das ligações entre amígdala e tálamo (S ouza, 1996; Rotrou, 1993). 
As respostas emocionais provavelmente se originem nas ligações entre amígdala e hipotálamo. Estas emoções, podem influir na aprendizagem, pois ativam outras ligações da amígdala para as vias sensoriais. Conseqüentemente a relação entre aprendizagem e memória podem estar ligadas às condições emocionais da pessoa (Retrou, 1993).

É freqüentemente observada na clínica de pessoas idosas que muitas das queixas da memória estão associadas a episódios emocionais traumatizantes ou no mínimo marcantes e, que na seqüência, a pessoa desenvolve uma alteração da memória. Como exemplo, podemos citar a depressão. Nesta patologia, muitas vezes, a queixa básica é déficit de memória. Ressalte-se para este fato, exemplo claro da dimensão afetiva interferindo na "performance" da memória.

Verifica-se que ao tratar a depressão, o indivíduo refere melhora da queixa básica de esquecimento e afasta com estes fatos, o medo da "perda da memória", relacionado ao processo demencial, o qual vem acompanhado de diminuição de automomia e dependência.

Relativo ao planejamento com papel importante na resolução de problemas e de acompanhamento, está o córtex pré-frontal, sua ligação com o lobo temporal e tálamo, fazem crer que ele esteja envolvido com a memória.

Esta distribuição "pan-encefálica" da memória nos permite formar uma concepção de que as imagens não são armazenadas de forma inteira, mas em fragmentos. Por exemplo: não existe um lugar específico no cérebro para armazenar a palavra cadeira. Vários conjuntos de neurônios podem ativar a evocação de uma palavra e serão diferentes neurônios, dependendo da palavra que se evoca. Para armazenar as lembranças o cérebro decompõe em elementos de base que se encadeiam ao infinito.

Da mesma maneira que, com as letras do alfabeto podemos formar uma quantidade imensa de palavras, ou que, com apenas sete notas musicais é possível compor infinitas melodias. A maneira de codificar e remanejar permanentemente as lembranças faz intervir a personalidade e a história de cada um. Cada indivíduo registra, armazena e remaneja as lembranças à sua maneira, construindo assim a sua própria personalidade. A memória é incessantemente revista e as lembranças, reclassificadas, em função do seu uso. Algumas lembranças são recalcadas, outras modificadas e outras freqüentemente utilizadas (Guillet, 1989).

0 cérebro humano tem diferentes tipos de memória que vem sendo estudados. Sabemos que a memória não esta localizada em uma estrutura isolada do cérebro: ela é um fenômeno biológico e psicológico, envolvendo uma aliança de sistemas que funcionam juntos. Acrescentaria a este conceito a dimensão social da vida do homem, em especial, da família, a qual contribui para a qualidade da memória. A memória é viva, percebe, trabalha, muda permanentemente (Ledanseur, 1996) Segundo Lussier et al (1991), a memória representa 0 conjunto das estruturas e dos processos cognitivos que permitem codificar, armazenar e recuperar diversos tipos de informações.

Este processo de armazenagem tem sido área de estudos com diferentes enfoques teóricos. A atenção é condição básica para que haja memorização. Um grau ótimo de atenção é absolutamente necessário para que se consolide uma lembrança. Atenção e motivação caminham juntas; dirige-se maior atenção para aquilo que é de interesse. A ativação cerebral é diretamente proporcional ao grau de interesse que se mantenha numa tarefa.

O sistema de armazenagem que foi descrito por Atkinsons e Shiffrim (1971), e utilizado até hoje, se divide em memória de curto prazo e memória de longo prazo, no que se refere às relações da memória com o tempo e o espaço.

Sistema de curto prazo retém temporariamente as informações e o sistema a longo prazo responsável pelo armazenamento por um período maior de tempo. Segundo Baddeley (1992), a memória a curto prazo é também conhecida como memória primária ou imediata, que é relativa a atividades imediatas ou de execução momentânea ou de fatos recentes. Esta se encontra alterada nos processos demenciais e, em especial, na demência do tipo Alzheimer. Por esta razão, as alterações na memória de curto prazo, mesmo que não sejam patológicas, são vivenciadas com grandes conflitos internos. Os esquecimentos habituais nas pessoas se não esclarecidos, podem levar a crer que trata-se do início de uma doença, fato este, que pode não ser verdadeiro.

A memória a longo prazo é também denominada de memória secundária ou funcional, ou para acontecimentos de passados distante. Esta memória permite a aquisição e a evocação de informações aprendidas.

0 processo de memorização funciona basicamente em dois tempos. Primeiramente, a mensagem é recebida e depois armazenada. Sabemos que no cérebro a armazenagem a curto prazo se processa, para em seguida ocorrer a armazenagem a longo prazo. Não é possível precisar exatamente o limite entre ambas. 
Se recebemos muitas mensagens ao mesmo tempo, sem nos darmos tempo para refletir, muitas informações serão perdidas.

A memória de fatos recentes, é aquela que se refere ao que aconteceu nos segundos, minutos, horas, dias, meses, anos próximos; e, a memória dos fatos antigos é a dos acontecimentos vividos na infância, na adolescência e nos primeiros anos da vida adulta. A memória de fatos recentes é mais frágil e vulnerável ao envelhecimento normal e patológico (Rotrou 1993).

$\mathrm{Na}$ memória existem diferentes processos de interação permanente entre órgãos dos sentidos que recebem a mensagem, certas zonas que armazenam as impressões, e outras que as analisam e as codificam. Cada órgão desempenha um papel fundamental no processo da memória. Assim dizemos: memória visual, memória auditiva, memória olfativa e memória táctil.

As informações que entram pelos órgãos dos sentidos em geral são combinadas, ou seja: ouvimos e vemos, ou ainda, vemos e sentimos 0 aroma. Atribui-se um significado ao que se percebe. A semântica e a memória sempre estão associadas, donde, se tem, que a memória semântica designa a lembrança dos acontecimentos culturais e dos conceitos. A linguagem possibilita que o pensamento seja expresso por meio da palavra. Esta pode ser escrita ou falada por um sistema de signos e símbolos. A memória verbal denota as relações entre as funções da memória e linguagem.

A outra função cognitiva que intervém para designar a aptidão da memória para estabelecer relações ou associações é a inteligência. Desta forma, se tem a memória lógica ou associativa. A inteligência desempenha um papel fundamental na estruturação e organização das informações. Os laços entre memória e inteligência vão mais longe e permite a representação mental do espaço, denominada memória viso-espacial.

Existe ainda a memória de procedimentos ou "procedural" que se entende por saber fazer; a memória prospectiva, que prevê o fato; a memória retrospectiva que é aquilo que foi feito; e a memória episódica ou declarativa, que se refere a evocação dos fatos ou acontecimentos vividos.

R elativo ao estudo das funções cognitivas e sua relação com o envelhecimento, este revela que a memória reflete a qualidade de vida e que não se perde a memória porque se perde células mas, porque ao envelhecer passamos a viver de maneira diferente. Desta forma fazemos um uso muito reduzido da memória deixando de estimulá-la e reduzindo as informações à serem processadas e armazenadas e, com isto, empobrecendo o seu conteúdo e fragilizando um recurso de bem viver.

Quanto aos sinais gerais do envelhecimento, estes tornam-se cada vez mais visíveis à medida em que o tempo cronológico avança. Estudos e pesquisas em Gerontologia demonstram que o envelhecimento entre os órgãos ocorre de forma assincrônica e difere de um indivíduo para outro (Cavalcanti, 1975). É um processo global e abrange os níveis biológico, psicológico e social.

Sinais evidentes de envelhecimento do sistema nervoso podem ser observados e identificados quando se nota, por exemplo:

\begin{tabular}{|c|c|c|c|c|c|c|}
\hline \multicolumn{7}{|c|}{ ALTERAÇÕES DOS ÓRGÃOS DOS SENTIDOS } \\
\hline SINAIS NEURO-OFTALMOLÓGICOS & 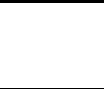 & & $\square$ & $\mathbb{U}$ & 孟菂 & $\square$ \\
\hline SINAIS NEURO-AUITIOS & IIIII & & & & 피 & \\
\hline $\begin{array}{l}\text { SINAIS NEURO-OLFATO- } \\
\text { GUSTATIOS }\end{array}$ & 四 & 盂 & $\square$ & $\square$ & & \\
\hline
\end{tabular}

\begin{tabular}{|c|c|}
\hline \multicolumn{2}{|r|}{ ALTERAÇÕES MOTORAS } \\
\hline SINAIS MOTORES & 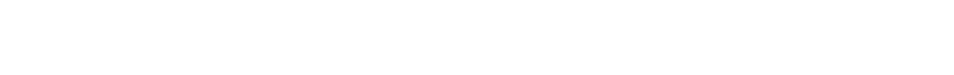 \\
\hline SINAIS RE $\square \square$ E $\square$ OS & 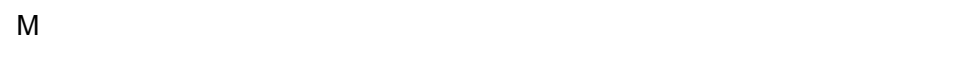 \\
\hline
\end{tabular}

Estes sinais são apenas indicativos do processo de envelhecimento normal de algumas das funções do sistema nervoso, os quais podem colaborar para a evolução da perda da autonomia. No entanto, sabe-se que se o potencial funcional existente continuar a ser estimulado, verifica-se pela observação clínica que algumas funções melhoram seu desempenho. 
Nenhuma das modificações acima citadas tem o mesmo peso quanto ao significado da mudança na "performance" da memória. "Perder a memória" equivale mais ou menos a sentir que "perde-se a cabeça", ser incapaz de manter a autonomia, de não saber o que esta acontecendo, de se tornar gradativamente dependente. Fatores sociais e afetivos, tais como: isolamento, solidão, empobrecimento afetivo e evidentemente quadros orgânicos patológicos podem levar o idoso a um processo demencial (Almeida, 1997).

Trabalhos desenvolvidos no sentido de motivar o idoso à participação em atividades que privilegiem a estimulação psicossocial tem revelado subjetiva e clínicamente a melhora da "performance da memória", com evidência de aumento da "auto-confiança", da "auto-estima", permitindo um crescente sentimento de autonomia, e por conseqüência, aumentando sua participação na vida ativa, maior capacidade para o auto-cuidado diminuindo os riscos da dependência.

A preocupação com a memória não pode ser um fenômeno de modismo ou apenas das pessoas que estão se tornando idosas, menos ainda, de preocupação apenas com as técnicas mnemônicas que existem desde a antigüidade. Mas, o que se torna importante na atualidade, é saber que, graças a técnicas de "visualização" no cérebro dos correspondentes neurobiológicos é possível saber o que se passa no cérebro quando este recebe um estímulo cognitivo graças ao desenvolvimento da neurofisiologia.

Estes procedimentos podem ser valorizados pela compreensão do conceito de "plasticidade cerebral", que indica a capacidade do cérebro de se modificar favoravelmente em sua estrutura e funcionamento, sob o efeito de estímulos apropriados e constantes. Isto se deve a eletrofisiologia.

A neurobiologia possibilitou a fundamentação científica de "programas de ativação cerebral", com base nos conhecimentos da plasticidade cerebral. Verifica-se que com aumento da atividade cerebral ocorre evidentemente aumento da atividade neuronal e das ramificações dendríticas e da eficiência sináptica.

Os Programas de Ativação Cerebral - PACs, desenvolvidos por R otrou (1993), obedecem princípios científicos da neurobiologia, pedagógicos e práticos. Outros autores também propõem "laboratórios de memória" com a intenção de possibilitar uma estimulação cognitiva para melhorar a "performance da memória", pois, hoje se admite que quanto mais as células cerebrais são estimuladas, maiores são as trocas físico-químicas e maiores as possibilidades dos neurônios serem preservados. Os neurônios não ativados ou em desuso, ou os "neurônios preguiçosos" tem maiores chances de se desconectarem e desaparecerem. Isto não significa que a ativação cerebral impeça que neurônios desapareçam ou que impeça patologias mentais. As pesquisas demonstram que os exercícios das funções cognitivas aumentam a eficácia das sinapses (Rotrou, 1993).

A estratégia de trabalho do programa de ativação da memória de Rotrou, pode ser desenvolvida de forma individual ou em grupo. Alguns autores apontam para os benefícios de laboratórios de memória serem desenvolvidos em grupo, pois permitem a interação com outras pessoas, 0 aprendizado de novas estratégias (Barbara Wilson; Nick Moffat ,1992). Yves Ledanseur (1994) propõe trabalhos em "oficinas de memória", no qual este autor valoriza bastante 0 aspecto psicossocial relacionados as queixas de memória.

Os idosos que participam destes trabalhos apresentam em geral queixa de esquecimento sem comprometimentos patológicos e podem ter um ótimo aproveitamento, desfazendo em grande parte a preocupação com a "perda da memória", a dependência e a diminuição da autonomia.

Estas práticas têm melhorado a autonomia e por conseguinte a relação dos idosos sobre o meio, as influências do meio sobre os idosos, caracterizando a melhora da independência, uma vez que os idosos passam a desenvolver suas atividades da vida diária e da vida prática com maior auto-confiança e auto-suficiência.

Para os idosos com comprometimento patológico da memória, principalmente os pacientes que apresentam algum tipo de demência, evidentemente, precisa ser dispensado tratamento diferenciado e principalmente garantir um aporte de apoio técnico-científico e psicológico para os familiares. Esta forma de apoio pode ser em atendimentos específicos aos familiares ou em grupos de auto-ajuda orientados por profissionais e familiares ,como é o caso de associações específicas existentes em nosso meio.

\section{CONCLUSÃO}

Não restam dúvidas de que os mecanismos da memória serão mais bem compreendidos, bem como os processos de envelhecimento cerebral mais aclarados, quando o desenvolvimento e o funcionamento do cérebro forem estudados com mais profundidade. 
Neste estudo, tentou-se fornecer algumas bases iniciais para a discussão sobre as queixas de memória e as relações com as dimensões biológicas normais e patológicas. As dimensões sociais e afetivas da alteração da memória e seus reflexos na autonomia do idoso acarretam implicações diretas na vida da família com necessidade de reorganização, muitas vezes, estruturais e profundas para todos os seus membros. Fenômeno que merece estudo aprofundado uma vez que constitui-se em fato novo, presente numa sociedade cada vez mais de "cabelos brancos" caracterizando-se também como dimensão gerontológica do envelhecimento populacional.

Nas alterações da memória muitas áreas não foram totalmente exploradas e aprofundadas; algumas funções cerebrais foram apenas mencionadas. Apesar disso, entende-se que fica evidenciado a importância de se considerar na queixa da memória, sua relação com o contexto sócio-familiar do indivíduo e as possibilidades de minimizar os efeitos psicossociais do déficit cognitivo, quando este for estimulado adequadamente.

A vida em família, a sua casa junto aos referenciais construídos ao longo da trajetória da sua vida, as relações com os próximos e os laços afetivos podem, em muito, minimizar sejam as alterações cognitivas, seja o curso de uma doença com perda irreversível da memória. É preciso que se fique atento à rede de suporte familiar.

ABSTRACT: The study of cognitive functions is an area in clear development in the field of neuroscience, and is also an area of general interest. Studies and research try to characterize cognitive problems and distinguish between what is normal and what is pathological. It is observed that many elders complain about memoryrelated difficulties, and some decline in the normal cognitive function can also be verified in the normal aging process - a fact that worries the elderly as well as his/her family, since it's normally associated to the idea of dependency. Thus, forgetfulness must be distinguished from the "memory loss" associated to demential processes. Sciences have been advancing towards that aim and a special contribution from neuropsychology is noteworthy. Studies have demonstrated the existence of disabling pathologies, accompanyed by a relevant deficit in the memory function, with loss of autonomy and independence. We must, however, be aware of relationship between an individual's memory and his/her biological, psychological, and social contexts. Memory is alive, it works an changes constantly.

KEY WORDS: Memory; Aging; Autonomy; Family.

\section{REFERÊNCIAS BIBLIOGRÁFICAS}

1. ALMEIDA, O. P. Manejo dos distúrbios de comportamento em pacientes demenciados. In: FORLENZA, O. V.; ALMEIDA, O. P. Depressão e demência no idoso. São Paulo : Lemos, 1997.

2. AMANCIO, A.; CAVALCANTI, P. C. U. Clínica geriátrica. Rio de J aneiro : Atheneu, 1975.

3. ATKINSON, R. C. SHIFFRIN, R. N. The control of short term memory. Scientific American, n.225, p.82-90, 1971.

4. BADDELEY, A. D. Memory theory and memory therapy. In: WILSON, B.; MOFFAT, N. Clinical management of memory problems. California : Singular, 1992.

5. BARBIZET, J ., DUIZABO, P. Manual de neuropsicologia. Porto Alegre : Artes Médicas, 1985.

6. BOTTINO, M. C. C., CID, C. G., CAMARGO, C. H. P. Avaliação neuropsicológica. In: FOR LE NZA, O. V.; ALMEIDA, O. P. Depressão e demência no idoso. São Paulo : Lemos, 1997.

7. CANINEU, P. R. O envelhecimento e as mudanças no sistema nervoso. O mundo da sáude, v.21, n.6, 1997.

8. FARIA, C. T. Envelhecimento In: JACOB, W. F. Envelhecimento do sistema nervoso e a dor no idoso. São Paulo : FMUSP, 1996.

9. FOLSTEIN, M. F.; FOLSTEIN, S. E.; MCHUGH, P.R. Mini mental state: a pratical method for grading the cognitive state of patients for clinican. J. Psychiatr. Res. n.12, p.189-198, 1975.

10. FONSECA, V. da. Introdução as dificuldades de aprendizagem. Porto Alegre : Artes Médicas, 1985.

11. FORETTE, F.; CHRISTEN, Y.; BOLLER, F. Plasticité cérébrale et stimulation cognitive. FNG. Paris, 1993.

12. GILMORE, Community surveys and mental health. In: ANDERSON, W., JUDLE, T. (Ed.). Geriatric medicine. N. Y. : Academic Press, 1974. 
13. GRAEFF, F. G.; BRANDÃO, M. L. Neurobiologia das doenças mentais. 4.ed. São Paulo : Lemos, 1997.

14. GUILLET, Pierre. L'aventure de I’age. Paris : Hatier, 1989.

15. GURLAND, B, CROSS, P. Epidemyology of psychopathology in old age. Psychiatr. Clin. North Am. v.5, n.11, 1982.

16. INGBERMAN, Y. K. Luria e distúrbios de aprendizagem. Trabalho apresentado na mesa redonda: Psicologia do Desenvolvimento: uma Visão Soviética - III Encontro Paranaense de Psicolologia. Curitiba, agosto 1989.

17. LEDANSEUR, Y. Guia del animador del taller memoria. Fundación Memória y Vida, Sucy en Brie, 1996.

18. LEGER, J . M.; TESSIER, J . F.; MOUTY, M. D. Psicopatologia do envelhecimento: assistência às pessoas idosas. Petrópolis: Vozes, 1996.

19. LEVY, R. Declínio cognitivo associado ao envelhecimento. In: ALMEIDA, O. P., NITRINI, R. (Org). Demência. São Paulo : Byk, 1995.

20. LÚRIA, A. R. Fundamentos de neuropsicologia. São Paulo : Universal, 1981.

21. LUSSIER, I. et al. Caracterisation des troubles de la mémoire dans la demence de type Alzheimer In: HABIB, M.; J OANNETLE, Y. et PUEL, M. (Ed.). Syndromes démentiels: approche clinique et neuropsychologique. Paris : Masson, 1991.

22. MENESES, M. S. TEIVE, H. A. G. Doença de Parkinson, aspectos clínicos e cirúrgicos. Rio de J aneiro : Guanabara Koogan, 1996.

23. ROTROU, J. de. La mémoire en pleine forme - comment l'éntretenir, la préserver, la développer. Paris : Robert Lafond, 1993.

24. SALAZAR, R. L. Gerontologia y geriatria. México : Intramericana, 1985.

25. SOUZA, R. R. Alterações anatômicas no sistema nervoso associadas ao envelhecimento In: J ACOB, W. F. Envelhecimento do sistema nervoso central e a dor no idoso. São Paulo : FMUSP, 1996.

26. TAR NOPEL, L. Crianças com distúrbio de aprendizagem. São Paulo : Edart, 1980.

27. VAN DER LINDER, M.; HUPET, M. Le vieillissement cognitif. Paris : PUF, 1994

28. VAN EERSEL, P. La source noir. Paris : Grosset, 1987.

29. WILSON, B.; MOFFAT, N. Clinical management of memory problems. California : Singular, 1992. 
